\title{
ALTOS DIRECTIVOS PÚBLICOS: UN NUEVO CONJUNTO DE DATOS DE MIEMBROS DEL SERVICIO CIVIL CHILENO*
}

\author{
Bastián González-Bustamante \\ University of Oxford, Reino Unido \\ Universidad de Santiago de Chile, Chile \\ bastian.gonzalezbustamante@politics.ox.ac.uk \\ Matías Astete \\ Universidad de Santiago de Chile, Chile \\ matias.astete@usach.cl \\ Berenice Orvenes \\ Universidad de Santiago de Chile, Chile \\ berenice.orvenes@usach.cl
}

\section{RESUMEN}

Este artículo presenta un conjunto de datos novedoso de altos directivos públicos chilenos durante el período 2009-2017. El foco de este artículo metodológico es demostrar cómo el uso de procesos de minería de datos y aprendizaje automático pueden ser útiles para la creación del conjunto de datos y sus potenciales aplicaciones. Primero, presentamos cómo creamos y validamos esta base. Luego, presentamos algunas aplicaciones descriptivas y estimaciones no-paramétricas de supervivencia con curvas KaplanMeier. Esperamos que este conjunto de datos sea un recurso relevante para profundizar la compresión del funcionamiento del servicio civil en Chile y realizar distintas comparaciones que permitan ampliar la línea de investigación sobre personal político y gubernamental.

Palabras clave: Servicio Civil, Alta Dirección Pública, Estado, Minería de Datos, Chile.

Esta investigación fue posible gracias al financiamiento de la Universidad de Santiago de Chile (USACH) bajo el Convenio de Desempeño Académico 2018 en el marco del proyecto "Survival of the Senior Civil Servants in the Chilean Executive Branch" (DOI: 10.17605/OSF.IO/WBF6M).

Revista de Gestión Pública | Volumen IX, Número 2 | Julio-Diciembre 2020 | issn 0719-1820 


\title{
SENIOR PUBLIC MANAGERS: A NOVEL DATASET ON MEMBERS OF THE CHILEAN CIVIL SERVICE
}

\begin{abstract}
This paper presents a novel dataset of senior public managers in Chile in 2009-2017. This methodological study focuses on demonstrating how data mining and machine learning can be useful for the creation of the dataset and its potential applications. We explain how we created and validated the dataset before going in to present some descriptive applications and nonparametric survival estimates with Kaplan-Meier curves. We hope that this dataset will serve as a relevant resource for gaining a deeper understanding of the Chilean civil service and making different comparisons through which to expand this research line on political and government personnel.
\end{abstract}

Keywords: Civil Service, Senior Public Management, State, Data Mining, Chile. 


\section{INTRODUCCIÓN}

Los sistemas de servicio civil se han consolidado y profesionalizado en la mayoría de los países del mundo durante el último siglo, situación que ha permitido que los Estados mejoren la provisión de bienes y servicios públicos (Raadschelders y Rutgers 1996). Naturalmente existe una gran heterogeneidad en estos procesos de consolidación al comparar entre países, sin embargo, se pueden advertir ciertos patrones. Por ejemplo, a comienzos del siglo XX existía una capacidad limitada de disponer de altos directivos públicos, lo que se cristalizó en una diferenciación entre la carrera funcionaria, o servicio civil ordinario, y la alta dirección pública (Longo 2003, González-Bustamante 2020, Martínez Puón 2012). Esta diferenciación, que en algunos países es más pronunciada e implica considerar de forma separada al cuerpo de altos directivos de los funcionarios comunes, ha sido un elemento crucial en las reformas de los servicios civiles latinoamericanos durante las últimas décadas (GonzálezBustamante 2018, Ramos y Scrollini 2013).

En efecto, es posible identificar procesos de profesionalización de la alta dirección pública y reformas cuyo fin es disminuir los grados de politización en los nombramientos de altos directivos durante las últimas décadas en América Latina (Cortázar, Lafuente y Schuster 2014). Este punto es particularmente sensible para practicantes y académicos que estudian el funcionamiento de la administración pública y la designación del personal gubernamental. Como indican Peters y Pierre (2004), altos grados de politización en los nombramientos se asocian con clientelismo político y patronazgo, lo que perjudica el equilibrio deseable entre designaciones de confianza política y sistemas de nombramientos basados en mérito.

Fue precisamente la sensación de un desbalance en aquel equilibrio lo que generó una ventana de oportunidad para instaurar el Sistema de Alta Dirección Pública (SADP) en Chile. Durante una profunda crisis política en 2003, producto de escándalos de corrupción, se realizó un acuerdo entre el gobierno y la oposición para impulsar una serie de iniciativas de modernización y transparencia para superar la crisis. Entre las medidas más relevantes se encontraban la regulación de las compras en el sector público y la creación del SADP (González-Bustamante, Olivares, Abarca y Molina 2016, Lambertini y Silva 2009). Sin embargo, este sistema tiene claroscuros.

El SADP ha sido destacado por organismos internacionales y practicantes como un caso exitoso en América Latina (González-Bustamante 2018, Iacovello y Strazza 2014). En términos generales es un modelo que 
aumenta la transparencia y los contrapesos institucionales (Pardow 2018, Pardow y Verdugo 2020). Sin embargo, existen algunos aspectos que requieren de una evaluación más precisa. Si bien durante la última década se constata una ampliación relevante en la cobertura del sistema, existe evidencia de elevados niveles rotación de los altos directivos y una baja permanencia en los servicios más críticos que están mandatados por ley a utilizarlo (González-Bustamante 2020). Aunque es posible suponer que lo segundo se solucionó, al menos parcialmente, con la reforma de 2016 que prohíbe los nombramientos provisionales, el problema de la rotación no ha sido estudiado en profundidad. En este contexto, es posible suponer que la ausencia de este tipo de trabajos se relaciona con la falta de datos sistematizados cuyo levantamiento requiere de un esfuerzo relevante de procesamiento.

Los costos y las dificultades asociadas al procesamiento de grandes volúmenes de información pueden ser superadas con el uso de técnicas de minería de datos e inteligencia artificial cuyas potencialidades, hasta ahora, no han sido del todo explotadas por las ciencias sociales latinoamericanas. En esta línea, este artículo, de carácter eminentemente metodológico, se enfoca principalmente en demostrar las potencialidades de la minería de datos y del aprendizaje automático para elaborar bases de datos sociales. En específico, presentamos un nuevo conjunto de datos que contiene información detallada de 431 altos directivos públicos del primer nivel jerárquico del servicio civil chileno durante el período 2009-2017². El conjunto de datos fue creado con los antecedentes de dos solicitudes de acceso a información pública realizadas a la Dirección Nacional del Servicio Civil (DNSC) en 2016 y 2018 y una revisión de 1.396 documentos públicos, principalmente decretos y noticias institucionales. Estos documentos fueron digitalizados con algoritmos de minería de datos y revisados de forma semi-automatizada exhaustivamente. Esto nos permitió obtener diversa información de los altos directivos públicos. Los datos y el código serán liberados bajo una licencia de acceso abierto ${ }^{2}$. De momento, explicamos cómo fueron construidos y validados con el objetivo de orientar el uso de estas técnicas en la investigación sobre servicio civil y personal gubernamental.

En los siguientes apartados presentamos cómo creamos y validamos este conjunto de datos. Posteriormente, se exponen algunas aplicaciones

1 Este nivel, como indican González-Bustamante, Olivares, Abarca y Molina (2016), está compuesto principalmente por jefes de servicios públicos. Es relevante destacar que el SADP no tiene alcance sobre los cargos de confianza política más altos en el poder ejecutivo como, por ejemplo, el gabinete y cargos en los gobiernos regionales.

2 Se ofrecen detalles sobre el proceso de liberación de datos y código de replicación en el apartado final. 
descriptivas que ofrecen algunas luces de cómo estos datos podrían ser utilizados para mejorar la comprensión del funcionamiento del servicio civil chileno. Finalmente, discutimos brevemente potenciales comparaciones y aplicaciones para entender las dinámicas propias de los servicios civiles y las tensiones entre sus grados de politización y la estabilidad gubernamental.

\section{CREACIÓN DEL CONJUNTO DE DATOS}

Las fuentes originales de información corresponden a datos liberados por la DNSC en respuesta a las solicitudes AE004T0000240 y AE004T0000484 en el marco de la Ley 20.285 sobre Acceso a la Información Pública, realizadas el 26 de diciembre de 2016 y el 26 de abril de 2018, respectivamente. Con la primera solicitud elaboramos una base de datos de altos directivos públicos para el período 2009-2015 ( $N=391$, véase también González-Bustamante 2020). Con esta base de datos y la segunda solicitud elaboramos un listado de 452 directivos del primer nivel jerárquico para el período 2009-2017. Posteriormente, recopilamos 1.396 documentos públicos, entre decretos de nombramiento, actas de los concursos, noticias institucionales, entre otros similares.

Los documentos fueron cargados en la plataforma Open Science Framework $(\mathrm{OSF})^{3}$ y se les asignó una URL permanente única que nos permitió aplicar un algoritmo de reconocimiento óptico (OCR) programado específicamente para este propósito. La aplicación de procesos reconocimiento óptico sigue varias etapas (Smith 2007). En primer lugar, se identifican componentes conectados y se anidan los contornos identificables y las líneas de texto en cada documento. Las líneas de texto, posteriormente, se dividen en palabras considerando los espacios entre estas. Luego, cada palabra se intenta reconocer con diccionarios de datos entrenados, lo que opera como una especie de línea base. Este proceso implica aprendizaje automático (machine learning), por tanto, se realiza una iteración con el fin de reconocer palabras que en una primera instancia no fueron identificadas. Finalmente, se revisan los espaciados difusos donde no hubo resultados concluyentes. Todo este procedimiento es computacionalmente costoso, sin embargo, gracias al aumento sostenido en las capacidades de procesamiento durante las últimas décadas se ha vuelto accesible. De esta forma, es posible realizar el reconocimiento óptico y obtener texto manejable para su análisis.

Este tipo de algoritmos tienen una serie de aplicaciones relevantes que han sido incorporadas principalmente en motores de búsqueda $y$

3 Software abierto que facilita la colaboración e investigación científica, particularmente la replicabilidad de los proyectos. 
en la administración de grandes volúmenes de datos no numéricos. Esta segunda aplicación tiene una serie de potencialidades en el marco de la gestión documental en organismos públicos y privados y, además, facilita la posterior aplicación de técnicas de aprendizaje automático y profundo en el contexto de enfoques de inteligencia artificial. Por ejemplo, es posible distinguir como potenciales aplicaciones de inteligencia artificial el apoyo a la gestión del conocimiento, la automatización de procesos, los sistemas autónomos con capacidad de aprendizaje, los sistemas de reconocimiento, entre otros (Salvador 2021, Wirtz, Weyerer y Geyer 2019).

En el caso de esta base, los documentos PDF fueron convertidos en imágenes PNG que fueron cargadas en el repositorio del proyecto en $\mathrm{GitHub}^{4}$ que está conectado con OSF. Luego, las imágenes se convirtieron a un formato de texto manejable a través del proceso previamente descrito con el fin de emparejar y verificar los documentos con los casos identificados. Esto nos permitió validar los casos. La Figura 1 presenta el diagrama del proceso de recolección de información, elaboración del conjunto de datos y su validación.

Con estos documentos reconstruimos la trayectoria de 431 casos, es decir, excluimos 21 individuos (4,65\%) del conjunto de datos final ya que no pudimos encontrar fuentes de respaldo confiables. Esto nos permitió construir diversas variables como sexo, edad estimada, número de identificación nacional ${ }^{5}$, profesión, ministerio, servicio público, cargo desempeñado, estatus de adscripción al SADP y del nombramiento ${ }^{6}$, fechas de nombramiento y término de funciones, renovación en el cargo y razón del término de funciones.

4 Plataforma para alojar proyectos con sistema de control de versiones que facilita el seguimiento de cambios en códigos programados. Es una excelente plataforma para garantizar replicabilidad de análisis y proyectos de investigación.

5 Con el número de identificación nacional calculamos el año de nacimiento estimado con el siguiente modelo lineal $Y=$ año $=\alpha+\beta *$ (número de identificación) $+\varepsilon$, donde $\alpha=1.924,246$ y $\beta=4,202$. Este modelo tiene un $R^{2}$ ajustado de 0,911 y se estimó con datos de miembros del Congreso chileno entre las legislaturas de 1990 y 2014 (véase González-Bustamante 2014). Finalmente, se estima la edad con el año de nacimiento y la fecha de nombramiento del alto directivo público. Obtuvimos siete casos $(1,62 \%)$ con edades estimadas menores a 25 ańos debido a que su número de identificación es muy elevado. Estos casos han sido codificados como perdidos.

6 La cobertura del SADP se ha ampliado más de un 140\% durante la última década y existen diversas agencias públicas que lo utilizan de forma voluntaria (GonzálezBustamante 2020). Respecto a los nombramientos, por otra parte, existió hasta la reforma de 2016 la posibilidad de realizar nombramientos transitorios que operaban en un bucle que configuraba un resquicio para evadir el uso del sistema y realizar nombramientos discrecionales en servicios que estaban mandatados a utilizar el sistema (González-Bustamante, Olivares, Abarca y Molina 2016). 
Figura 1: Diagrama del proceso de recolección de información, elaboración del conjunto de datos y su validación

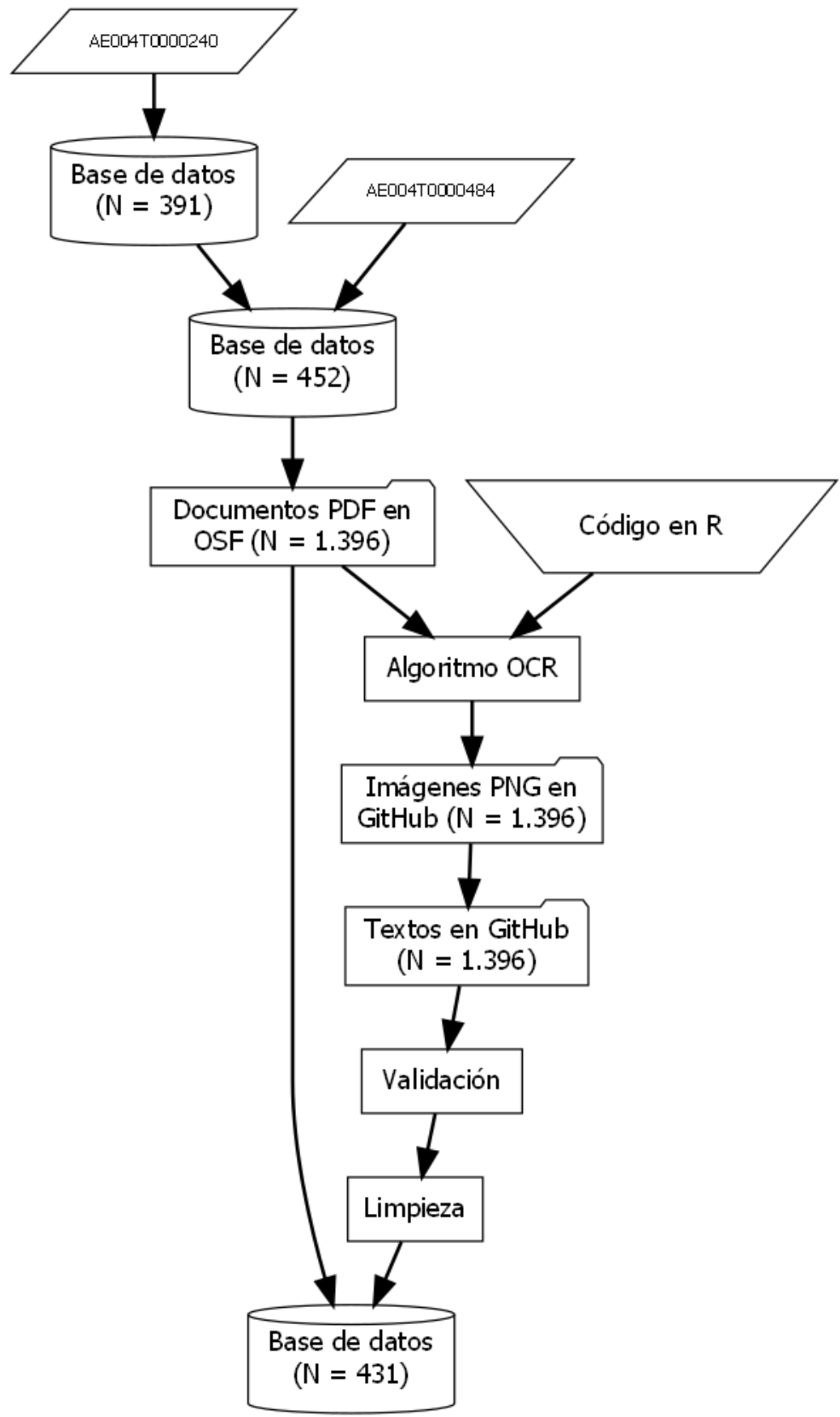

Fuente: Elaboración propia.

Es relevante destacar que este tipo de aplicaciones y sus potencialidades son particularmente relevantes para enfrentar los desafíos que tienen las 
administraciones públicas de Iberoamérica. La Declaración de Lisboa de 2020, por ejemplo, reconoce la necesidad de potenciar el uso de big data y la inteligencia artificial para predecir, analizar y evaluar la prestación de servicios públicos en la región (CLAD 2021). En efecto, la inteligencia artificial y la aplicación de distintos algoritmos dan cuenta de una nueva etapa de interacción entre las tecnologías de información, gobiernos y administraciones públicas, que se caracteriza principalmente por la creciente disponibilidad de grandes volúmenes de datos y la existencia de nuevas dinámicas en las prestaciones de servicios públicos (Criado 2021).

\section{VALIDACIÓN Y ANONIMIZACIÓN}

El proceso de digitalización con OCR se dividió en tres rondas. Primero, se excluyeron 62 documentos ya que corresponden a actas de concursos y los individuos no pudieron ser identificados con ninguna combinación lógica de sus nombres y apellidos. Posteriormente, se aplicó el algoritmo programado en 1.334 documentos con un éxito del 85,53\%. De los 193 documentos en los que el algoritmo falló, en 186 archivos fue por problemas de acentos y de tipeo que fueron corregidos con un código de limpieza. Este procedimiento aumentó el éxito a un 99,48\%. En consecuencia, el algoritmo solo falló en la identificación de seis documentos $(0,45 \%)$ y solo un documento $(0,08 \%)$ fue mal catalogado por nuestro equipo de investigación.

A continuación, la Figura 2, presenta una evaluación de la precisión del algoritmo de reconocimiento óptico revisando la proporción de texto que logró identificar correctamente. Para esto se extraen las palabras más comunes del español y se contrastan con diccionarios de palabras del idioma utilizados para entrenar los modelos Long Short Term Memory (LSTM) usados por Tesseract OCR Engine. En el contexto de los procesos de aprendizaje profundo, las redes neuronales permiten combinar procesos en iteraciones, por tanto, cada bucle considera información de las redes previas. Dependiendo del caso, algunas aplicaciones pueden requerir información reciente o datos más antiguos. Los modelos LSTM son redes neuronales que realizan el proceso de aprendizaje incluso si la brecha entre la información requerida y el punto de requerimiento es alta, por tanto, pueden recordar información después de varias iteraciones gracias al uso de diversas capas neuronales (Kumar, Goomer y Singh 2018). Por otra parte, Tesseract es un motor para reconocimiento óptico que se comenzó a desarrollar en 1995 en Bristol y desde 2005 está disponible como un código abierto (Smith 2007). Actualmente es usado por Google y ofrece soporte Unicode (UTF-8) para más de 100 idiomas a través de diversos lenguajes de programación (Ooms 2019, véase también el repositorio en 
GitHub https://github.com/tesseract-ocr/tesseract). Nuestros resultados muestran que, en general, la precisión se encuentra sobre el 90\%.

Figura 2: Evaluación del desempeño y precisión del algoritmo OCR

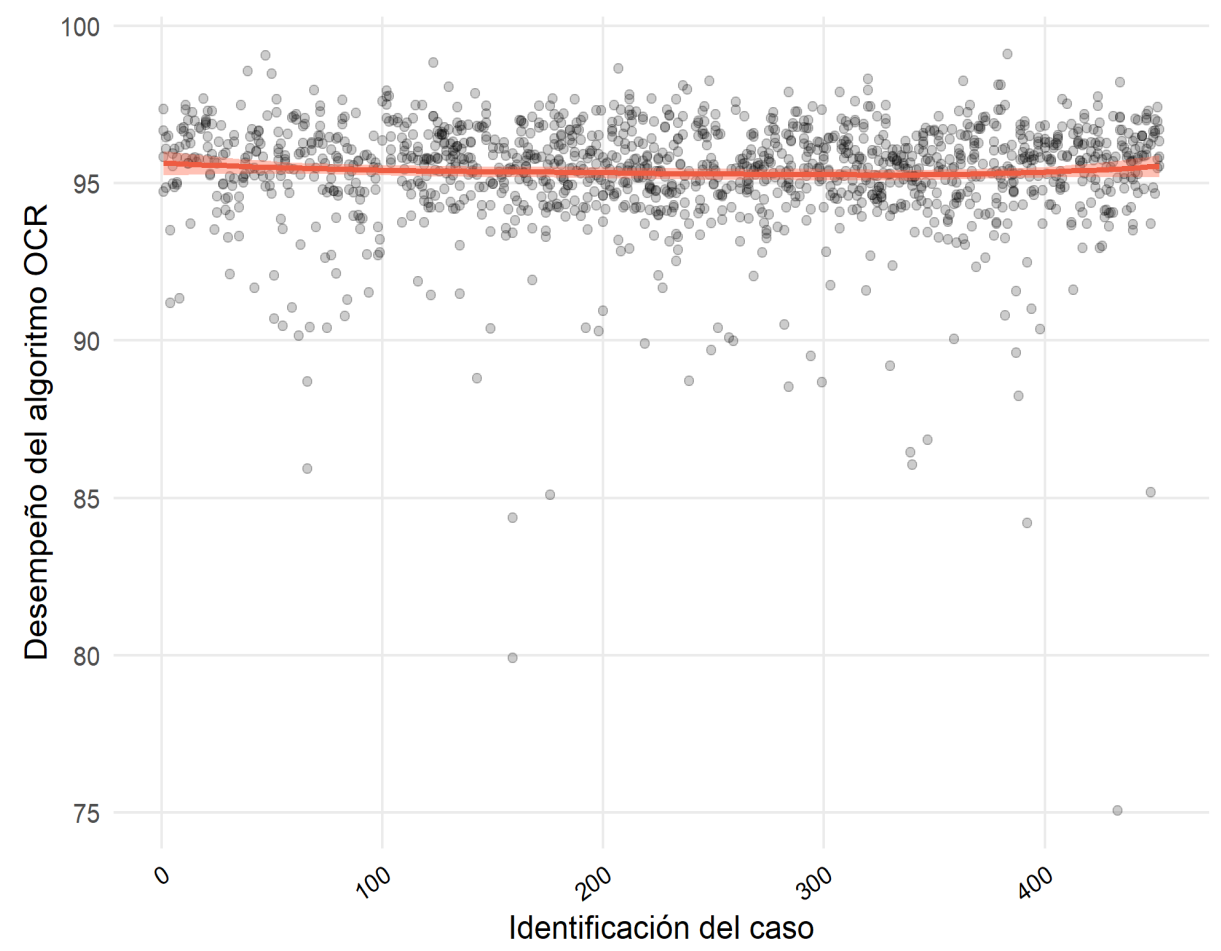

Fuente: Elaboración propia.

Además, revisamos de forma automatizada la variable sexo usando el primer nombre de cada caso y una base de datos de nombres de diversos países del mundo, sexo y su predicción estimada creada por Casper Strömgren en 2013 (véase Wais 2016). Esta base crece diariamente con datos extraídos de perfiles de redes sociales y en el momento de la revisión contaba con 111.541.298 observaciones, de las cuales 210.959 (0,19\%) correspondían a casos de Chile específicamente. Revisamos nuestra base inicial $(N=$ 453), proceso que implicó ejecutar 39.851.141 revisiones (Figura 3). Esto permitió identificar seis casos $(1,33 \%)$ con problemas de clasificación, de los cuales tres $(0,66 \%)$ es por el uso de un nombre que en otros países está asociado al sexo opuesto, por tanto, se limitó la validación al conjunto de datos de Chile $(n=210.959)$. Otros tres casos presentaban problemas por tener más de dos nombres, en consecuencia, se limitó la combinación lógica de nombres y se rastreó el más común. 
Solo dos casos $(0,44 \%)$ estaban efectivamente mal codificados y fueron corregidos manualmente. Con una inspección visual de la Figura 3 se pueden identificar cuatro casos con probabilidades inferiores al $75 \%$, al revisar los segundos nombres estas observaciones se validan elevando su probabilidad de 0,57 a 0,97 , de 0,67 a 0,98 , de 0,68 a 0,99 , y de 0,68 a 0,97 , respectivamente.

Figura 3: Validación de la variable sexo

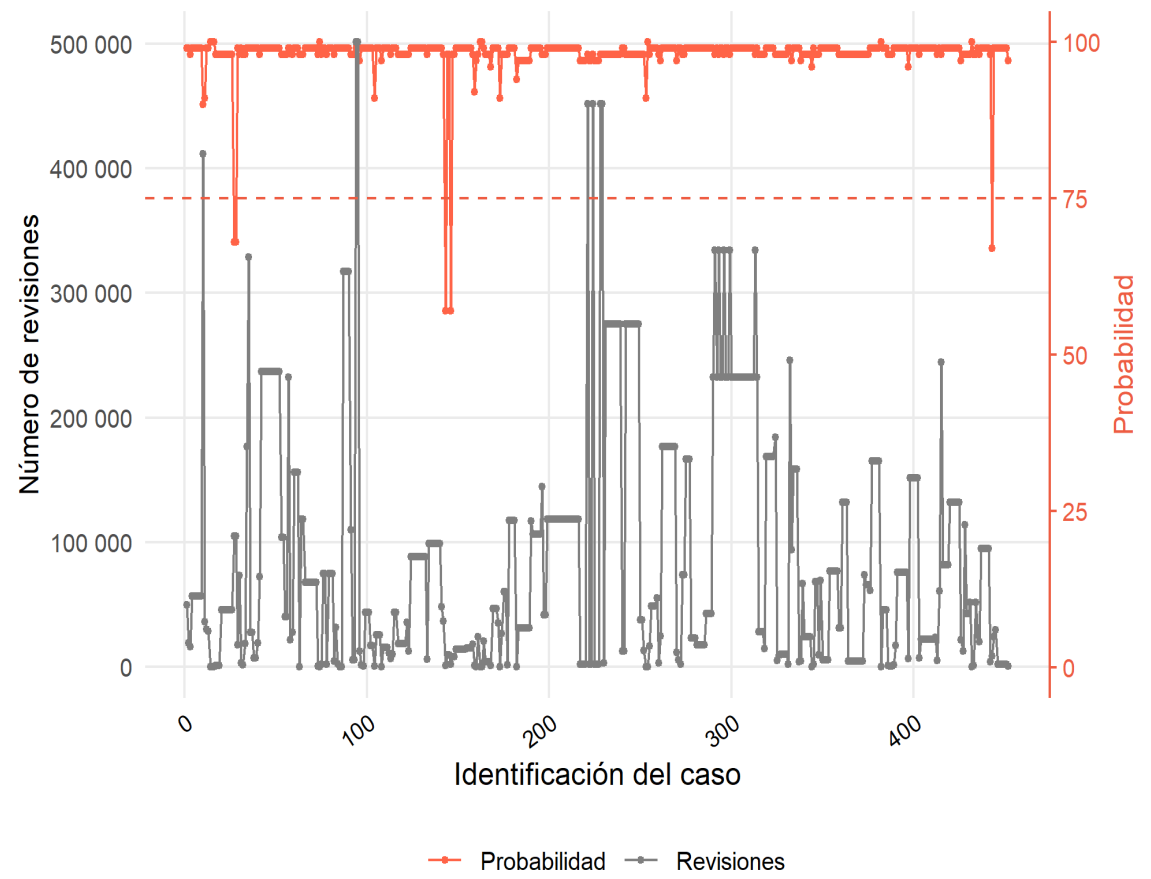

Fuente: Elaboración propia.

Por último, las variables que contienen información personal, como el nombre o el número de identificación nacional del individuo, fueron anonimizadas en la versión final del conjunto de datos con un algoritmo criptográfico basado en una función hash que bloquea la ingeniería-reversa, en consecuencia, imposibilita el acceso a los datos originales (Shinder y Cross 2008). Utilizamos específicamente Secure Hash Algorithm (SHA) de una longitud de 256 caracteres (SHA256) por razones de seguridad: los algoritmos de menor extensión son susceptibles a ser vulnerados, aunque desde los 128 bits el nivel de seguridad ya es bastante elevado (Eddelbuettel 2020). 


\section{APLICACIONES}

A continuación, presentamos algunos breves resultados exploratorios que podrían profundizarse al realizar cruces considerando variables como la profesión de los altos directivos o información más detallada de las instituciones donde se desempeñan. Posteriormente, en la sección final, se presentan unas breves reflexiones para potenciales comparaciones con otros datos existentes y futuras aplicaciones.

En primer lugar, la Figura 4 presenta frecuencias simples de algunas variables discretas del conjunto, específicamente del tipo de salida del cargo, sexo, estatus de afiliación del organismo al SADP e información sobre cuántos altos directivos fueron renovados en su puesto por un período adicional y aquellos que fueron nombrados discrecionalmente con nombramientos transitorios.

Figura 4: Frecuencias de las variables tipo de salida del cargo, sexo, afiliación del organismo, renovación y nombramientos transitorios

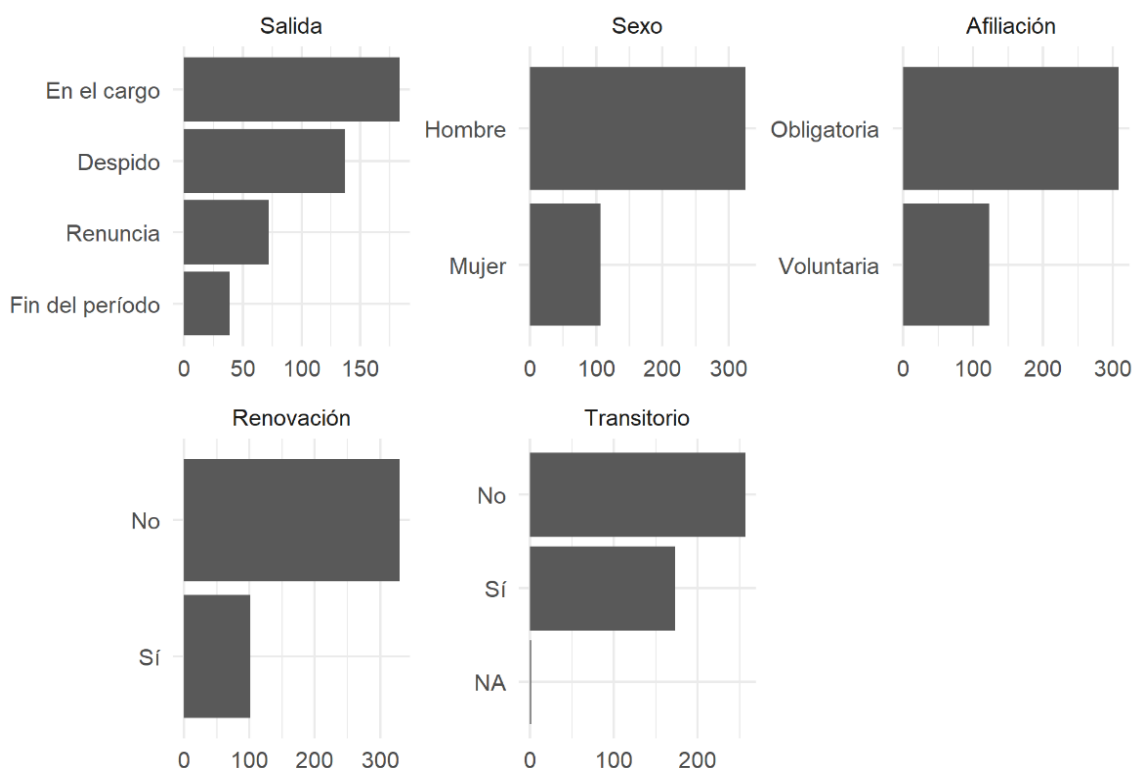

Fuente: Elaboración propia.

La muestra está compuesta mayoritariamente por hombres, tan solo el $24,59 \%$ son mujeres, y solo un pequeño grupo recibió renovación de su nombramiento $(23,67 \%)$, mientras que un número mayor fue nombrado de forma transitoria $(40,14 \%)$. Por otra parte, los trasfondos educacionales 
más recurrentes son estudios en derecho $(23,90 \%)$, medicina $(16,47 \%)$ o negocios y economía $(13,92 \%)$. Otro dato interesante es la cantidad de altos directivos despedidos durante su período $(31,79 \%)$ y los que renunciaron voluntariamente $(16,71 \%)$. La Figura 5, por otro lado, muestra la distribución de la edad estimada al momento del nombramiento del directivo y su tiempo de permanencia en el cargo en días.

Figura 5: Frecuencias de las variables edad estimada y tiempo de permanencia en el cargo

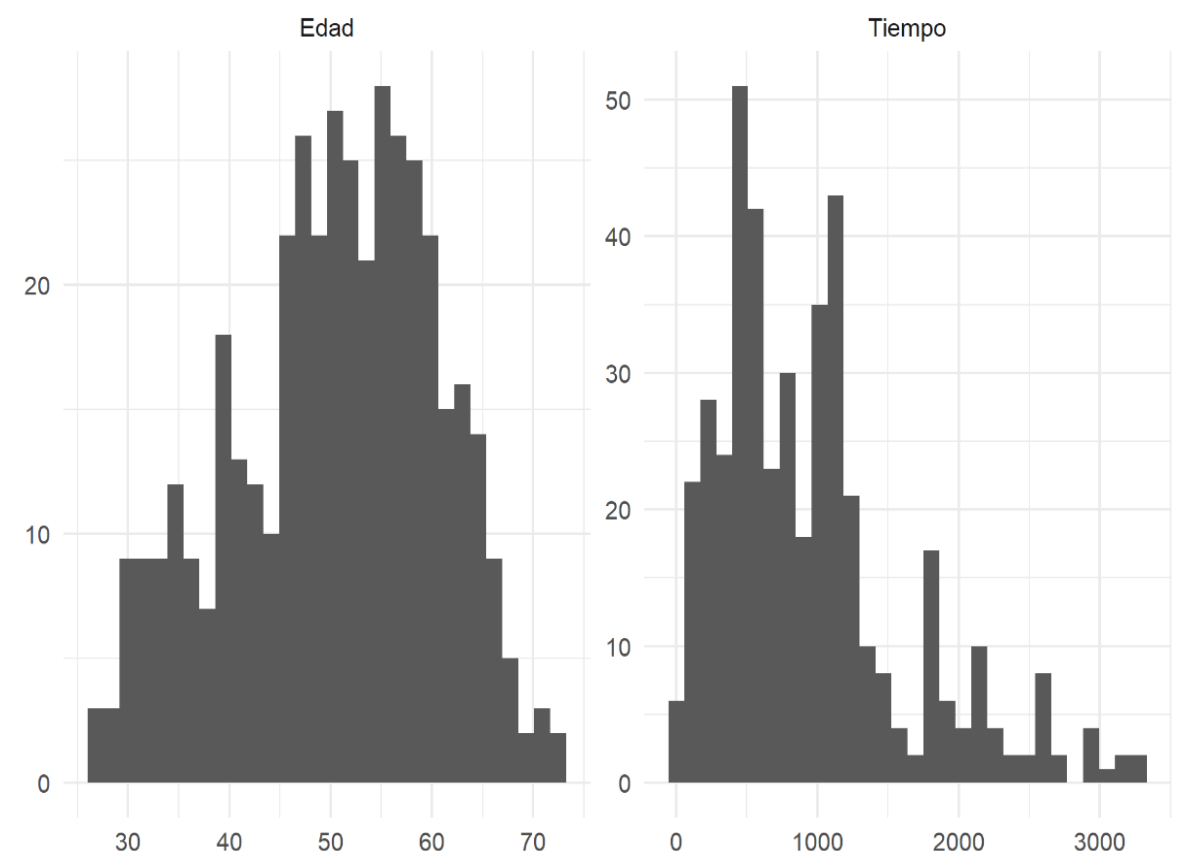

Nota: Se indica edad estimada al momento del nombramiento y permanencia en dias.

Fuente: Elaboración propia.

A continuación, realizamos algunas estimaciones no-paramétricas de supervivencia. Nuestro período de estudio $T$ corresponde período 20092017. En este período es posible observar la ocurrencia de diversos posibles eventos de interés como renuncias voluntarias, despidos, fin de períodos o altos directivos que a fines de diciembre de 2017 seguían en su cargo. Para esta aplicación nos interesan los despidos, por tanto, asumimos los otros resultados como ceros suponiendo la ausencia de riesgos competitivos. De esta forma, siguiendo a Austin, Lee y Fine (2016), a partir de , donde S(t) representa la función de supervivencia, es posible estimar la incidencia del 
despedido de altos directivos durante $T$. Una de las formas más directas de calcular la función de supervivencia en ausencia de riesgos competitivos son las estimaciones Kaplan-Meier, las cuales se calculan considerando el número de individuos al inicio del período y aquellos que van experimentando el evento de interés sobre el número inicial (Austin, Lee y Fine 2016, Kishore, Goel y Khanna 2010). A continuación, presentamos tres estimaciones Kaplan-Meier: por sexo, directivos que se desempeñan en el sector salud y aquellos que dirigen instituciones públicas del sector educación (Figura 6).

Figura 6: Estimaciones de supervivencia Kaplan-Meier para altos directivos públicos por sexo y sectores salud y educación

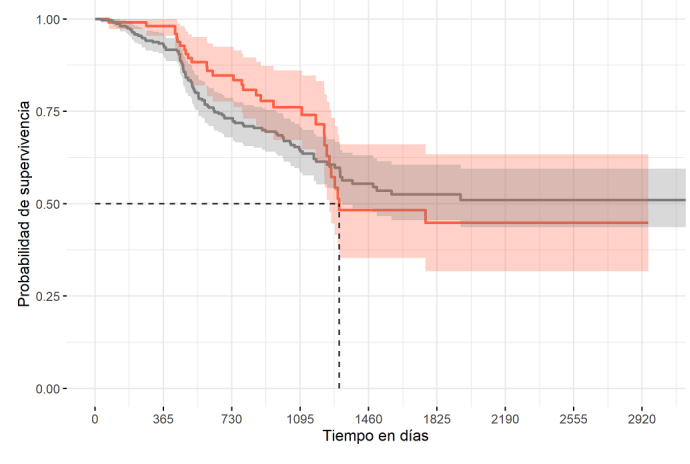

- Mujer - Hombre
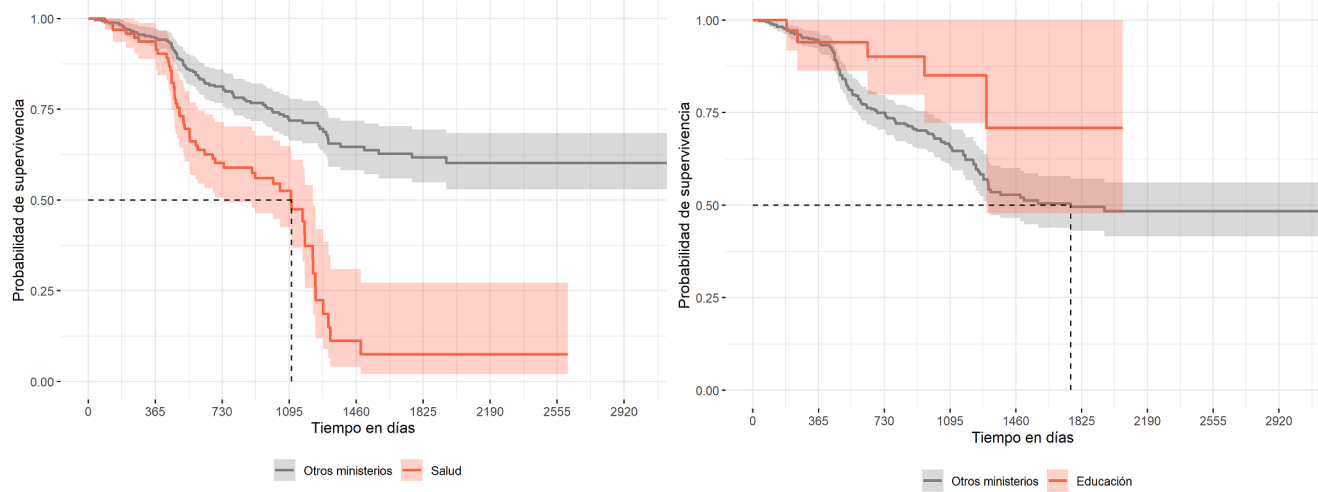

Nota: Se gráfica intervalo de confianza del 95\% y la supervivencia media. Si bien la estimación considera el tiempo en días, las rupturas del eje X permiten visualizar intervalos que corresponden a años. Fuente: Elaboración propia.

De estas estimaciones la única diferencia estadísticamente significativa es que los directivos que trabajan en servicios de salud muestran una supervivencia bastante más baja en el cargo. Por otra parte, aquellos que se 
desempeñan en el sector educación tienden a sobrevivir más en sus cargos, aunque el resultado no es tan robusto como se aprecia al examinar los intervalos de confianza. Sorpresivamente no hay diferencias significativas entre hombres y mujeres, sin embargo, es importante considerar que el número de directivas en la muestra es bajo y que los datos están desbalanceados, por tanto, es necesario aplicar estrategias de identificación causal en la estimación econométrica para obtener hallazgos robustos sin sesgo.

\section{OBSERVACIONES FINALES}

Este artículo muestra que la aplicación de algoritmos de reconocimiento permite automatizar ciertos procesos y facilitar la administración de grandes volúmenes de información. Esto es útil para académicos y practicantes pues constituye uno de los principales desafíos para las administraciones públicas Iberoamericanas en el contexto de las nuevas potencialidades que ofrece la inteligencia artificial en el marco de la gestión pública. Además, este trabajo es un ejemplo sobre cómo las leyes de acceso a la información pueden facilitar la investigación cuando se combinan con innovaciones tecnológicas y procesos de inteligencia artificial.

El conjunto de datos permanecerá bajo embargo hasta la segunda mitad de 2022. Posteriormente, será liberado en formato de valores delimitados por comas con codificación UTF-8. Los materiales complementarios se pueden revisar en el proyecto en OSF (DOI: 10.17605/OSF.IO/WBF6M). El código de replicación se encuentra disponible en nuestro repositorio de GitHub por ahora privado y próximamente disponible para consulta pública.

Este conjunto de datos es potencialmente comparable con otros que ya existen sobre élites políticas y personal gubernamental en Chile. Por una parte, es posible utilizar la encuesta de élites usada por Joignant, Perelló y Torres (2015) que cubre distintas posiciones entre 1990 y 20107. Por otro lado, es posible realizar comparaciones con los datos de gabinetes a nivel ministerial de González-Bustamante y Olivares (2016, 2018) y de subsecretarías de González-Bustamante y Olivares (2015). También es posible realizar comparaciones con los datos de puerta giratoria en el poder ejecutivo chileno de Maillet, González-Bustamante y Olivares (2019) o de las comisiones asesores presidenciales de Cisternas y Vásquez (2018). Por último, otra alternativa es utilizar información biográfica de candidatos al Congreso como González-Bustamante y Cisternas (2016).

$7 \quad$ Para detalles del muestreo véase el anexo sobre estudios empíricos de élites y personal gubernamental en Chile de Maillet, González-Bustamante y Olivares (2016). 
Una segunda línea que es posible explorar tiene relación con incorporar datos de los organismos públicos de los altos directivos para diseñar estrategias empíricas más elaboradas. En este sentido, es relevante destacar que este conjunto de datos corresponde a una muestra no probabilística con datos observacionales, en consecuencia, cualquier estimación econométrica que se realice debería ser complementada con estrategias de identificación causal que permitan solucionar la ausencia de contrafactuales y controlar posibles sesgos adecuadamente. 


\section{REFERENCIAS}

Austin, P. C., Lee, D. S. y Fine, J. P. (2016). Introduction to the Analysis of Survival Data in the Presence of Competing Risks. Circulation, 133 (6), 601-609.

Cisternas, C. y Vásquez, J. (2018). Comisiones Asesoras Presidenciales en Chile: Entre la expertise y la pluralidad de actores sociales. European Review of Latin American and Caribbean Studies, 106, 1-24.

Consejo Latinoamericano de Administración para el Desarrollo (CLAD) (2021). Inteligencia artificial y ética en la gestión pública. Caracas: CLAD.

Cortázar, J. C., Lafuente, M. y Schuster, C. (2014). Strategies to Modernize the Civil Service in Latin America. En Cortázar, J. C., Lafuente, M. y Sanginés, M. (Eds.), Serving Citizens: A Decade of Civil Service Reforms in Latin America (2004-13). Washington D. C.: IDB.

Criado, J. I. (2021). Inteligencia artificial: madurez tecnológica, adopción e innovaciones en la gestión pública. En Inteligencia artificial y ética en la gestión pública. Caracas: CLAD.

Eddelbuettel, D. (2020). Create Compact Hash Digests of R Objects [Package]. Debian.

González-Bustamante, B. (2014). Elección directa de consejeros regionales 2013. Rendimiento del capital político, familiar y económico en una nueva arena electoral en Chile. Política, Revista de Ciencia Politica, 52 (2), 49-91.

. (2018). Civil Service Models in Latin America. En Farazmand, A. (Ed.), Global Encyclopedia of Public Administration, Public Policy, and Governance. Cham: Springer.

. (2020). The Politics-Administration Dichotomy: A Case Study of the Chilean Executive during the Democratic PostTransition. Bulletin of Latin American Research, 39 (5), 582-597.

González-Bustamante, B. y Cisternas, C. (2016). Élites políticas en el poder legislativo chileno: La Cámara de Diputados (1990-2014). Politica, Revista de Ciencia Política, 54 (1), 19-52. 
González-Bustamante, B. y Olivares, A. (2015). Rotación de subsecretarios en Chile. Una exploración de la segunda línea gubernamental (19902014). Revista de Gestión Pública, IV (2), 151-190.

. (2016). Cambios de gabinete y supervivencia de los ministros en Chile durante los gobiernos de la Concertación (19902010). Colombia Internacional, 87, 81-108.

- (2018). La élite política gubernamental en Chile: Supervivencia de ministros. En Codato, A. y Espinoza, F. (Eds.), Las élites en las Américas: Diferentes perspectivas. Curitiba: Editora Universidade Federal do Paraná.

González-Bustamante, B., Olivares, A., Abarca, P. y Molina, E. (2016). Servicio civil en Chile, análisis de los directivos de primer nivel jerárquico (2003-13). Revista de Administração Pública, 50 (1), 59-79.

Iacoviello, M. y Strazza, M. (2014). Diagnostic of the Civil Service in Latin America. En Cortázar, J. C., Lafuente, M. y Sanginés, M. (Eds.), Serving Citizens: A Decade of Civil Service Reforms in Latin America (2004-13). Washington D. C.: IDB.

Joignant, A., Perelló, L. y Torres, J. (2015). Political Capital and the Unequal Career Origins of the Political Elite in Chile. En Dubrow, J. K. (Ed.), Political Inequality in an Age of Democracy. Cross-National Perspectives. Nueva York: Routledge.

Kishore, J., Goel, M. y Khanna, P. (2010). Understanding survival analysis: Kaplan-Meier estimate. International Journal of Ayurveda Research, 1 (4), 274-278.

Kumar, J., Goomer, R. y Singh, A. K. (2018). Long Short Term Memory Recurrent Neural Network (LSTM-RNN) Based Workload Forecasting Model For Cloud Datacenters. Procedia Computer Science, 125, 676682.

Lambertini, G. y Silva, E. (2009). Hacia un perfeccionamiento del Sistema de Alta Dirección Pública. En Tomicic, V. y García, C. (Eds.), Un mejor Estado para Chile: Propuestas de modernización reforma. Santiago: Consorcio para la Reforma del Estado.

Longo, F. (2003). La reforma del empleo público en las democracias avanzadas: Mérito con flexibilidad. En Echebarría, K. (Ed.), Servicio 
civil: Temas para un diálogo. Washington D. C.: IDB.

Maillet, A., González-Bustamante, B. y Olivares, A. (2016). ¿Puerta giratoria? Análisis de la circulación público-privada en Chile (20002014) (Working Paper No 7). Programa de las Naciones Unidas para el Desarrollo.

Maillet, A., González-Bustamante, B. y Olivares, A. (2019). Public-Private Circulation and the Revolving Door in the Chilean Executive Branch (2000-2014). Latin American Business Review, 20 (4), 367-387.

Martínez Puón, R. (2012). Hacia la profesionalización de la función directiva en Latinoamérica. Revista de Gestión Pública, I (2), 387-419.

Ooms, J. (2019). Open Source OCR Engine [Package]. Berkeley Institute for Data Science.

Pardow, D. G. (2018). ¿Técnicos o políticos?: Radiografía del sistema de nombramiento de directivos en agencias regulatorias independientes. Revista Chilena de Derecho, 45 (3), 745-769.

Pardow, D. G. y Verdugo, S. (2020). Estabilidad, profesionalismo y politización en los nombramientos de directivos de agencias reguladoras independientes: Lecciones desde la experiencia chilena. Revista Derecho del Estado, 46, 223-252.

Peters, B. G. y Pierre, J. (2004). Politicization of the Civil Service: Concepts, Causes, Consequences. En B. G. Peters \& J. Pierre (Eds.), Politicization of the Civil Service in Comparative Perspective: The Quest for Control. Nueva York: Routledge.

Raadschelders, J. C. N. y Rutgers, M. R. (1996). The Evolution of Civil Service Systems. En Bekke, H. A. G. M., Perry, J. L. y Toonen, T. A. J. (Eds.), Civil Service Systems in Comparative Perspective. Bloomington: Indiana University Press.

Ramos, C. R. y Scrollini, F. (2013). Los nuevos acuerdos entre políticos y servidores públicos en la alta dirección pública en Chile y Uruguay. Revista Uruguaya de Ciencia Politica, 22 (1), 11-36.

Salvador, M. (2021). Capacidades institucionales para afrontar las infraestructuras organizativa y ética de la inteligencia artificial en las administraciones públicas. En Inteligencia artificial y ética en la gestión 
pública. Caracas: CLAD.

Shinder, L. y Cross, M. (2008). Understanding Cybercrime Prevention. En Cross, M. (Ed.), Scene of the Cybercrime. Burlington: Elsevier.

Smith, R. (2007). An Overview of the Tesseract OCR Engine. Ninth International Conference on Document Analysis and Recognition (ICDAR 2007) Vol 2.

Wais, K. (2016). Gender Prediction Methods Based on First Names with genderizeR. The R Journal, 8(1), 17-37.

Wirtz, B. W., Weyerer, J. C. y Geyer, C. (2019). Artificial Intelligence and the Public Sector-Applications and Challenges. International Journal of Public Administration, 42 (7), 596-615.

Recibido: 01-10-2020

Aceptación de la versión final: 01-12-2020 